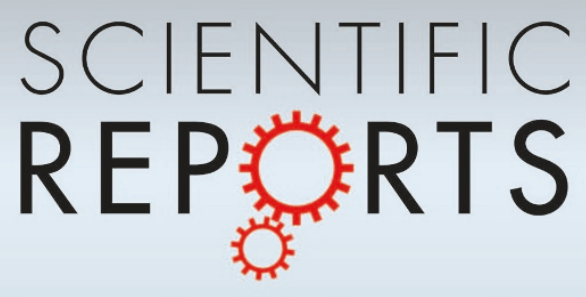

OPEN

SUBJECT AREAS:

CARBON NANOTUBES

AND FULLERENES

SYNTHESIS OF GRAPHENE

Received

15 April 2013

Accepted

16 July 2013

Published

5 August 2013

Correspondence and requests for materials should be addressed to G.E. (eresg@ornl.gov) or Z.W.P. (panz@uga.

edu)

\title{
Self-Assembly of Graphene on Carbon Nanotube Surfaces
}

\author{
Kaiyuan Li ${ }^{1,2}$, Gyula Eres ${ }^{3}$, Jane Howe ${ }^{3}$, Yen-Jun Chuang ${ }^{1}$, Xufan Li ${ }^{1}$, Zhanjun Gu ${ }^{1,4}$, Litong Zhang ${ }^{2}$, \\ Sishen $\mathrm{Xie}^{5}$ \& Zhengwei Pan'
}

${ }^{1}$ College of Engineering and Department of Physics and Astronomy, University of Georgia, Athens, GA 30602, USA, ${ }^{2}$ Science and Technology on Thermostructural Composite Materials Laboratory, Northwestern Polytechnic University, Xi'an, Shaanxi 710072, China, ${ }^{3}$ Materials Science and Technology Division, Oak Ridge National Laboratory, Oak Ridge, TN 37831, USA, ${ }^{4}$ Laboratory for Bio-Environmental Effects of Nanomaterials and Nanosafety, Institute of High Energy Physics, Chinese Academy of Sciences, Beijing 100049, China, Institute of Physics, Chinese Academy of Sciences, Beijing 100080, China.

The rolling up of a graphene sheet into a tube is a standard visualization tool for illustrating carbon nanotube (CNT) formation. However, the actual processes of rolling up graphene sheets into CNTs in laboratory syntheses have never been demonstrated. Here we report conformal growth of graphene by carbon self-assembly on single-wall and multi-wall CNTs using chemical vapor deposition (CVD) of methane without the presence of metal catalysts. The new graphene layers roll up into seamless coaxial cylinders encapsulating the existing CNTs, but their adhesion to the primary CNTs is weak due to the existence of lattice misorientation. Our study shows that graphene nucleation and growth by self-assembly of carbon on the inactive carbon basal plane of CNTs occurs by a new mechanism that is markedly different from epitaxial growth on metal surfaces, opening up the possibility of graphene growth on many other non-metal substrates by simple methane CVD.

\footnotetext{
raphene, a two dimensional (2D) single atomic layer of $s p^{2}$ bonded carbon, is the basic building block of all graphitic materials including $0 \mathrm{D}$ fullerenes, $1 \mathrm{D}$ carbon nanotubes (CNTs), and 3D graphite. The formation of these graphitic structures is customarily illustrated by either rolling up or stacking graphene sheets $^{1,2}$, and the inverse processes have been experimentally demonstrated by unzipping $\mathrm{CNTs}^{3,4}$ or exfoliating (un-stacking) graphite ${ }^{5,6}$. However, the actual processes of rolling up or stacking single layer graphene in laboratory synthesis to form larger graphitic structures have never been demonstrated.

The preference of the single layer graphene patches to assemble into larger structures is the basis for all carbon nanostructure formation and it is the key to controlling the size, structure and properties of CNTs by synthesis. In practice, the number of cylindrical graphene layers in a CNT, also referred to as "walls", is governed and determined at the time of formation by the growth parameters of a particular synthesis technique. Previous studies state that once formed, the diameter of an individual CNT remains unchanged regardless of how long the growth lasts (e.g., up to $48 \mathrm{~h})^{7}$ or how many times the growth process is repeated (e.g., up to 8 times) ${ }^{8}$. A number of attempts have been made to thicken existing CNTs by the deposition of pyrolytic carbon using chemical vapor deposition (CVD) processes ${ }^{9-11}$. Although thickening of the CNTs has been observed to occur, the deposited pyrocarbon was either amorphous or turbostratic. These, and similar results, are in agreement with thermodynamic calculations which predict that the curvature of a layer strongly influences its graphitizability. In particular, layers of less than $15 \mathrm{~nm}$ radius of curvature are believed to be non-graphitizable,

Here we report that we succeeded in conformal growth of fully graphitized graphene layers by carbon selfassembly on single-wall CNTs (SWCNTs) and multi-wall CNTs (MWCNTs) using CVD of methane. The process is referred to as self-assembly because unlike in CVD growth of CNTs no metal catalyst is necessary. The new graphene layers roll up into seamless coaxial cylinders, which encapsulate the existing CNTs. The present study shows that high-quality graphene layer growth is possible without strict registry with surface atoms. This process is distinct from the epitaxial growth of graphene on metal surfaces ${ }^{13-19}$ that is believed to require nearly an exact match with the metal lattice, opening up the possibility of graphene growth on a larger variety of substrates including many non-metal materials.
}

\section{Results}

SWCNTs with diameters of $\sim 1.5 \mathrm{~nm}$ (Fig. 1a) produced by arc-discharge ${ }^{20}$ and MWCNTs with diameters of 15-20 nm (inset of Fig. 2a) synthesized by CVD ${ }^{21}$ were used as the substrates for graphene growth. The CVD 

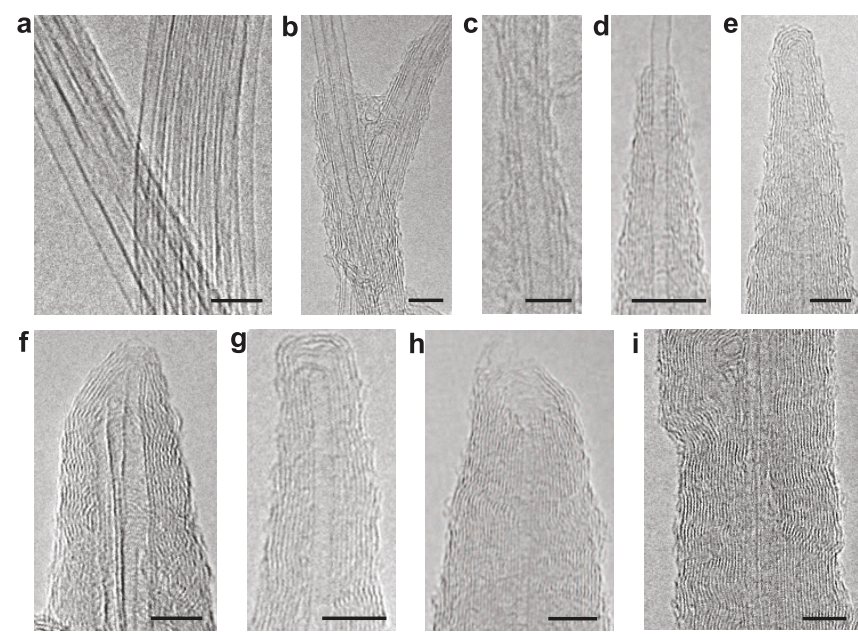

Figure $1 \mid$ High-resolution TEM images of graphene layers grown on SWCNTs. (a), Pristine SWCNT bundles prior to graphene growth. (b), (c), 1-3 layers of graphene grown locally on SWCNT surfaces. (d), A thickened CNT with a cone-shaped morphology. (e-h), Thickened nanotubes with different cap morphologies. The tube in (f) contains two SWCNTs in the core. There is a small gap in the cap of the tube in $(\mathrm{g})$. The cap of the tube in (h) is open. (i), A section of a thickened nanotube with two SWCNTs in the core. The growth times for the thickened nanotubes in (b) through (i) are $3 \mathrm{~min}(\mathrm{~b}, \mathrm{c}), 5 \mathrm{~min}(\mathrm{~d}), 8 \mathrm{~min}(\mathrm{e}-\mathrm{g})$, and $10 \mathrm{~min}(\mathrm{~h}, \mathrm{i})$. The scale bars are $5 \mathrm{~nm}$.

growth of graphene on the inactive basal planes of CNTs requires a growth temperature exceeding $1100^{\circ} \mathrm{C}$. Below $1100^{\circ} \mathrm{C}$ no graphene can be grown. In the present study, the deposition temperature was held constant at $1150^{\circ} \mathrm{C}$.

The graphene growth on a SWCNT paper containing micron size cracks (Supplementary Fig. S1a-b) was performed in a CVD tube furnace. The growth of graphene on SWCNTs depends critically on the deposition time. For deposition time shorter than 2 min, very few graphene layers were observed. After $3 \mathrm{~min}$ of deposition, 1-3 graphene layers form locally on individual or bundled SWCNT surfaces as shown by the high-resolution transmission electron microscopy (TEM) images in Figs. $1 \mathrm{~b}$ and $1 \mathrm{c}$. The graphene layers are wellaligned and concentric with the SWCNT walls. The TEM imaging shows growth occurring in two directions: radial growth that determines the diameter and wall number, and axial growth that extends the lateral size and closes each open shell into a cylinder. Axial growth along the free active edges is much faster; this, combined with radial growth to deposit new layers contiunoulsy onto the existing graphene surfaces, results in the formation of a cone-shaped MWCNT as shown in Fig. 1d (for more images see Supplementary Fig. S2a-c). When the growing graphene edges reach the SWCNT tip, they curve to form a cap around the tip. Figures 1e through $1 \mathrm{~h}$ illustrate that cap formation occurs regardless of whether the tip was originally open or closed and whether it is an individual CNT or a bundle of tubes (for more images see Supplementary Fig. S2d-g). The caps are generally fully closed (Fig. 1e-g); however, incompletely closed caps occasionally also appear (Fig. 1h). The graphene layers in the caps are typically close packed, but small gaps between layers can form (Fig. 1g; also Supplementary Fig. S2e) when the outer layers over shoot the inner ones. Since the growth rate at the active graphene edges (axial growth) is much faster than that on the inactive basal planes (radial growth), the new layers are completed rapidly, leading to the transformation of a SWCNT into a thick and uniform MWCNT after 10 min of growth, as shown in Fig. 1i.

The MWCNTs used for graphene growth are aligned nanotube sheets that were cleaved from a 6-mm-tall vertically aligned nanotube forest, as the scanning electron microscopy (SEM) images show
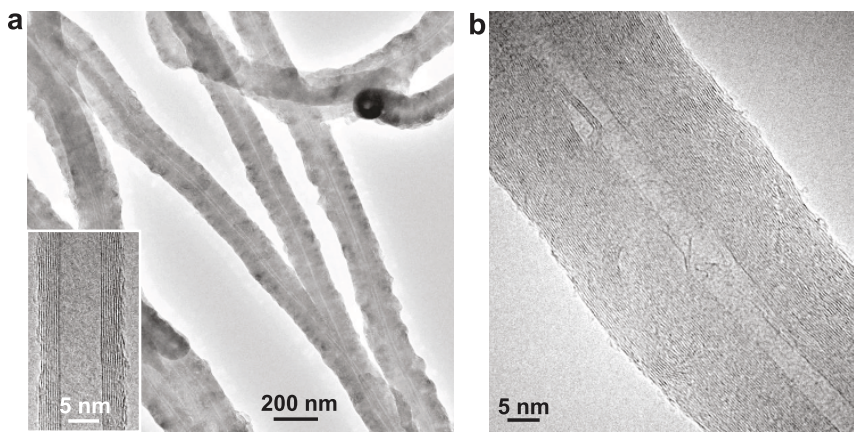

c
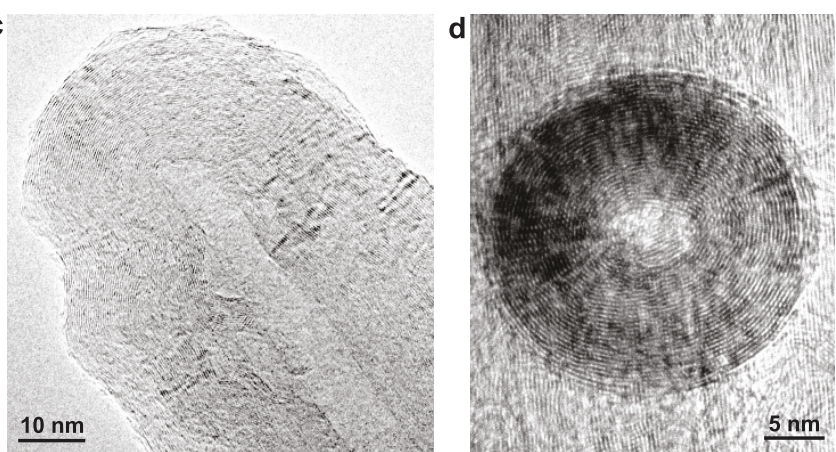

Figure $2 \mid$ TEM images of graphene grown on MWCNTs. (a), Lowmagnification TEM image of several thickened MWCNTs. The inset shows a high-resolution TEM image of a pristine MWCNT prior to graphene growth. (b), A section of a thickened tube. (c), The tip region of a thickened nanotube with a closed cap. (d), Top view of a thickened nanotube. The growth times for these thickened nanotubes are $60 \mathrm{~min}$ (a), $15 \mathrm{~min}$ (b), $30 \mathrm{~min}(\mathrm{c})$, and $20 \mathrm{~min}(\mathrm{~d})$.

in Supplementary Fig. S3. Figure 2a shows that after the deposition, the primary nanotubes are uniformly thickened along their entire length independent of their curvature (diameter) and the deposition time (see also Supplementary Fig. S4). The newly deposited graphene layers are concentric with respect to the primary tube axis (Fig. 2b$2 \mathrm{~d})$. The growth rate of graphene on MWCNTs is nearly constant over time with the diameters of the thickened tubes increasing to $\sim 20-30 \mathrm{~nm}, \sim 60-80 \mathrm{~nm}, \sim 100-150 \mathrm{~nm}$, and $\sim 200-250 \mathrm{~nm}$ after $10,30,60$, and $120 \mathrm{~min}$ of growth, respectively (Supplementary Fig. S4), corresponding to $\sim 2 \mathrm{~nm} / \mathrm{min}$ radial growth rate. After 60 $120 \mathrm{~min}$ of growth, the thickened tubes in the nanotube sheets start merging and coalescing to form a solid nanotube "block", as the SEM images shown in Supplementary Fig. S4d-4f. Since all the thickened tubes are well-graphitized and highly aligned, the solid nanotube block is a new carbon structure that is expected to have highly anisotropic physical properties in the directions along and perpendicular to the primary CNT axis, which are useful for novel electrical or thermal conductivity applications of carbon.

The high-resolution TEM images in Fig. 1 and Fig. 2 clearly show a full integration of the new graphene layers with the existing nanotube walls. However, because of the complete graphitization of the new walls the resulting composite structure appears indistinguishable from typical MWCNTs. An obvious proof of new wall formation is the transformation of the starting SWCNTs before growth in Fig. 1a to the resulting MWCNTs after growth in Fig. 1b-1i. The addition of new walls to existing MWCNTs was confirmed by the increase of diameters and wall numbers after growth compared to that before growth (Fig. 2a). The crystallinity of the new walls was assessed qualitatively by comparison of image characteristics with the primary CNT walls present in the same image. Although the new walls are not identifiable by a difference in inter shell spacing in TEM images, the adhesion between the graphene coating and the primary nanotubes is found to be weaker than the inter wall interactions in the 
primary MWCNTs. The intentional fracturing of the thickened tubes reveals that the graphene coating breaks easier than the primary nanotubes, as shown by the pullout of the primary nanotubes in Fig. 3. TEM images in Supplementary Fig. S5 and SEM images in Supplementary Fig. S1d, Fig. S4b, and Fig. S4d-f provide further illustration of the pullout phenomenon.

Although the exact mechanisms that determine the stability of MWCNTs are still not fully understood, various models predict that there is an energetically favorable combination for the chirality of the nearest neighbor shells in a MWCNT ${ }^{22,23}$. The difference in the chirality of neighboring walls is the result of rotational misorientation of the respective honeycomb lattices, which directly affects the extent of $\pi$-stacking interaction between two adjacent layers. Such misorientation is highly prevalent in few layer graphene, complicating the understanding of the electronic properties ${ }^{24}$, and is found to be affected strongly by the growth conditions and preparation methods. In the present case, we attribute the pullout to a rotational misalignment (chirality difference) that exists first between the honeycomb lattices of the primary CNT and the new layers, and second between the adjacent lattices of the new layers. The existence of the lattice misorientation weakens interface bonding and allows the separation and slippage of the primary nanotubes from the new graphene layers under mechanical fracture.

\section{Discussion}

The most important implication of this work is related to understanding whether the self-assembly of carbon is influenced by the underlying hexagonal network of the CNTs, a method referred to as epitaxy in graphene growth literature. The concept of epitaxy is instrumental in CVD graphene growth that is performed on metal foils (including $\mathrm{Cu}$ and $\mathrm{Ni}$ ) where it is believed that a sufficiently
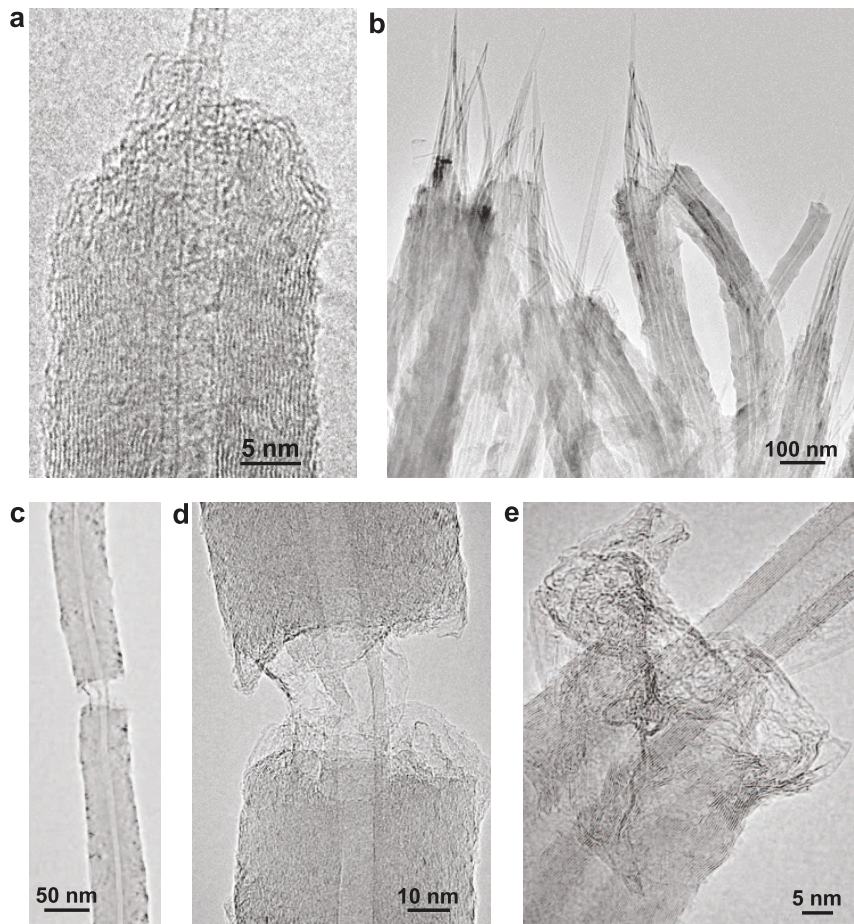

Figure 3 TEM images of fractured nanotubes. (a), A fractured nanotube showing the pullout of two primary SWCNTs. (b), Several fractured nanotubes showing the pullout of the primary MWCNTs. (c), Lowmagnification TEM image showing the fractured graphene segments connected by the primary MWCNT. (d), High-magnification TEM image of the broken part in (c). (e), A fractured nanotube formed by thickening two closely contacted MWCNTs. The images of torn and wrinkled graphene sheets are clearly visible in (a), (d), and (e). strong interaction between the carbon and the metal atoms facilitates close registry between the two lattices ${ }^{13-19}$. However, the current data are inconclusive on whether true registry between the carbon atoms of graphene and the metal lattice is prerequisite for the nucleation and the growth of graphene on all metals, in particular on copper.

In addition to lattice registry in graphene growth, the metal in CVD growth also performs a catalytic function in decomposition of the hydrocarbon molecules. The absence of metal in present work is a significant departure from currently used graphene growth processes and implies that a catalytic component is not indispensable in graphene growth. Instead, at sufficiently high temperatures, carbon self-assembles into a hexagonal network on the surface of the CNTs. The exact details of such a process and the deposition mechanisms of pyrocarbon in a CVD environment have been debated intensely for several decades and numerous models have been proposed ${ }^{25}$. According to existing literature, the most plausible mechanism for graphitized carbon deposition involves two distinct steps: a nucleation step and a growth step. The observations in the present study, particularly the cone-shaped nanotubes shown in Fig. 1a-1c with partially completed outer shells that represent the intermediate stages in this growth process, strongly support this two-step carbon deposition mechanism.

According to the mechanism, the nucleation step is proceeded by formation of polycyclic aromatic hydrocarbons (PAHs) either as liquid hydrocarbon droplets ${ }^{11,26}$ or as large gaseous PAH molecules $^{27,28}$. Since no droplets or oily residue were found on the thickened nanotubes, we attribute the formation of grapehen nucleation sites to large gaseous PAH molecules that preferentially form at high temperature ${ }^{28}$. This is also supported by our experimental observation that graphene grows on CNT surfaces only when the temperature exceeds $1100^{\circ} \mathrm{C}$. Each new CNT shell starts by nucleation of graphene on the inactive basal plane of a CNT initiated by physisorption of a large PAH molecule from the gas phase. Because of the high deposition temperature used in the present growth, the chirality between the new graphene nucleation patch and the existing carbon basal plane is likely to become random ${ }^{29}$, resulting in the formation of lattice misorientation. In the subsequent growth step, hydrocarbon species of any size are chemisorbed from the gas phase at the active graphene edges to feed the fast lateral growth of the graphene layers. When the graphene patch becomes sufficiently large, even before it is fully wrapped around and closed into a CNT, a new graphene patch can nucleate on top of it to continue this layer-by-layer like addition of new CNT walls. It is worth noting that our observations and the two-step carbon deposition mechanism explain the absence of radial growth during CVD of CNTs, which is typically carried out at temperatures well below $1100^{\circ} \mathrm{C}^{7,8}$.

In this study we discuss the conformal coating of CNT surfaces by graphene sheets using methane CVD. Our results demonstrates that the molecular interactions between successive $s p^{2}$ carbon layers that hold together the cylindrical sheets in CNTs and the planar sheets in graphite strongly depend on the growth parameters, particularly the temperature. Understanding these interactions is particularly significant for graphene growth on substrates other than the metals. The specific example of graphene growth on carbon basal planes in the parameter space studied in this work reveals that the adhesion between the newly deposited graphene layer and the substrate can potentially be manipulated by the preparation method. In addition to facilitating easy removal of the newly grown graphene layers by simple mechanical fracturing or chemical exfoliation, the existence of weak adhesion suggests that a strict epitaxial relationship is not necessary for the assembly of carbon into graphene, which opens up the possibility of graphene growth on many other non-metal substrates including such technologically important materials as $\mathrm{SiO}_{2}$. This work has great significance in several areas related to carbon nanostructure synthesis and applications including a new perspective on the mechanisms of graphene and MWCNT growth and the 
potential for using CNT walls as movable elements in nanoelectromechanical systems ${ }^{30}$.

\section{Methods}

Materials synthesis. We used SWCNT papers and aligned MWCNT sheets as the substrates for graphene growth. The SWCNT papers were prepared by ultrasonically dispersing SWCNT soot (in addition to containing a majority of SWCNTs, the soot also contains amorphous carbon, carbon nanoparticles and catalyst particles) fabricated by arc-discharge $e^{20}$ in alcohol followed by filtering and drying. The drying process created many micrometer size cracks in the papers, resulting in the exposure of individual and bundled SWCNTs as shown in Supplementary Fig. S1a-b. The after growth TEM characterization was focused on these exposed SWCNTs. The aligned MWCNT sheets were obtained by cleaving from a sidewall of a 6-mm-tall vertically aligned MWCNT forest ${ }^{21,31}$ using an adhesive tape (Supplementary Fig. S3). The thickness of the MWCNT sheets is about $10-20 \mu \mathrm{m}$. The SWCNT paper and MWCNT sheets were fixed vertically on a graphite beam that was then inserted in a horizontal tube furnace for subsequent growth of graphene. The typical growth experiments were performed at a furnace temperature of $1150^{\circ} \mathrm{C}$, methane flow rate of 5 standard cubic centimeters per minute (sccm) for the growth on SWCNTs or 10 $\mathrm{sccm}$ for the growth on MWCNTs, Ar flow rate of $150 \mathrm{sccm}$ at a chamber pressure of 150 Torr, and a deposition time of 1 to $10 \mathrm{~min}$ for the growth on SWCNTs or 1 to 120 min for the growth on MWCNTs. After the growth, the shapes of the SWCNT paper and MWCNT sheets were perfectly preserved as shown by Supplementary Fig. S1c-d for SWCNT paper and Supplementary Fig. S4 for MWCNT sheets.

Characterization methods. The morphology and structure of the samples were characterized by SEM (FEI Inspect F FEG SEM) and TEM (Hitachi HF-3300 FEG TEM/STEM). For TEM characterization, the as-grown SWCNT papers or MWCNT sheets were placed in between a folded copper TEM grid without damaging the integrity of the papers or sheets. Some papers and sheets were intentionally broken using tweezers for imaging the fractured surfaces.

1. Hamada, S., Sawada, S. \& Oshiyama, A. New one-dimensional conductors: Graphitic microtubules. Phys. Rev. Lett. 68, 1579-1581 (1992).

2. Dresselhaus, M. S., Dresselhaus, G. \& Eklund, P. C. Science of Fullerenes and Carbon Nanotubes: Their Properties and Applications (Academic Press, 1996).

3. Kosynkin, D. V. et al. Longitudinal unzipping of carbon nanotubes to form graphene nanoribbons. Nature 458, 872-876 (2009).

4. Jiao, L. Y., Zhang, L., Wang, X. R., Diankov, G. \& Dai, H. J. Narrow graphene nanoribbons from carbon nanotubes. Nature 458, 877-880 (2009).

5. Novoselov, K. S. et al. Electric field effect in atomically thin carbon films. Science 306, 666-669 (2004)

6. Hernandez, Y. et al. High-yield production of graphite by liquid-phase exfoliation of graphite. Nature Nanotech. 3, 563-568 (2008).

7. Pan, Z. W. et al. Very long carbon nanotubes. Nature 394, 631-632 (1998).

8. Li, X. S., Cao, A. Y., Jung, Y. J., Vajtai, R. \& Ajayan, P. M. Bottom-up growth of carbon nanotube multilayers: unprecedented growth. Nano Lett. 5, 1997-2000 (2005)

9. Endo, M. et al. Stacking nature of graphene layers in carbon nanotubes and nanofibers. J. Phys. Chem. Solids 58, 1707-1712 (1997).

10. Allouche, H. \& Monthioux, M. Chemical vapor deposition of pyrolytic carbon on carbon nanotubes. Part 2. Texture and structure. Carbon 43, 1265-1278 (2005).

11. Monthioux, M., Allouche, H. \& Jacobsen, R. L. Chemical vapor deposition of pyrolytic carbon on carbon nanotubes. Part 3. Growth mechanisms. Carbon 44, 3183-3194 (2006).

12. Speck, J. S., Endo, M. \& Dresselhaus, M. S. Structure and intercalation of thin benzene derived carbon fibers. J. Crystal Growth 94, 834-848 (1989).

13. Sutter, P. W., Flege, J. I. \& Sutter, E. A. Epitaxial graphene on ruthenium. Nature Mater. 7, 406-411 (2008).

14. Coraux, J., N'Diaye, A. T., Busse, C. \& Michely, T. Structural coherency of graphene on $\operatorname{Ir}(111)$. Nano Lett. 8, 565-570 (2008).

15. Kim, K. S. et al. Large-scale pattern growth of graphene films for stretchable transparent electrodes. Nature 457, 706-710 (2009).

16. Reina, A. et al. Large area, few-layer graphene films on arbitrary substrates by chemical vapor deposition. Nano Lett. 9, 30-35 (2009).
17. Chen, Z. P. et al. Three-dimensional flexible and conductive interconnected graphene networks grown by chemical vapor deposition. Nature Mater. 10, 424-428 (2011)

18. Li, X. S. et al. Large-area synthesis of high-quality and uniform graphene films on copper foils. Science 324, 1312-1314 (2009).

19. Bae, S. et al. Roll-to-roll production of 30-inch graphene films for transparent electrodes. Nature Nanotech. 5, 574-578 (2010).

20. Chang, B. H. et al. Loosely-entangled carbon nanotubes prepared by modified arcdischarge. J. Mater. Sci. Lett. 17, 1015-1017 (1998).

21. Eres, G., Puretzky, A. A., Geohegan, D. B. \& Cui, H. In situ control of the catalyst efficiency in chemical vapor deposition of vertically aligned carbon nanotubes on predeposited metal catalyst films. Appl. Phys. Lett. 84, 1759-1761 (2004).

22. Bellarosa, L., Bakalis, E., Melle-Franco, M. \& Zerbetto, F. Interactions in concentric carbon nanotubes: the radius vs the chirality angle contributions. Nano Lett. 6, 1950-1954 (2006).

23. Tison, Y., Giusca, C. E., Sloan, J. \& Silva, S. R. Registry-induced electronic superstructure in double-walled carbon nanotubes, associated with the interaction between two graphene-like monolayers. ACS Nano 2, 2113-2120 (2008).

24. MacDonald, A. H. \& Bistritzer, R. Graphene moiré mystery solved? Nature 474, 453-454 (2011).

25. Oberlin, A. Pyrocarbons. Carbon 40, 7-24 (2002)

26. Grisdale, R. O. The formation of black carbon. J. Appl. Phys. 24, 1082-1091 (1953).

27. Diefendorf, R. J. The deposition of pyrolytic graphite. J. Chim. Phys. 57, 815-821 (1960).

28. Hu, Z. J. \& Hüttinger, K. J. Mechanisms of carbon deposition - a kinetic approach. Carbon 40, 624-628 (2002).

29. Guo, W. L. \& Guo, Y. F. Energy optimum chiralities of carbon nanotubes. J. Am. Chem. Soc. 129, 2730-2731 (2007).

30. Popov, A. M., Lozovik, Y. E. \& Krivorotov, E. K. Can barrier to relative sliding of carbon nanotube walls be measured? Comput. Mater. Sci. 53, 67-74 (2012).

31. Gu, Z. J. et al. Aligned carbon nanotube-reinforced silicon carbide composites produced by chemical vapor infiltration. Carbon 49, 2475-2482 (2011).

\section{Acknowledgements}

Z.W.P. acknowledges funding by U.S. NSF (CAREER DMR-0955908). K.Y.L. thanks the financial support from the China Scholarship Council. G.E. acknowledges funding by the Materials Sciences and Engineering Division, Office of Basic Energy Science, U.S. Department of Energy. Z.J.G. acknowledges support by the National Basic Research Programs of China (973 program, No. 2012CB932504). The microscopy work was sponsored by Oak Ridge National Laboratory's Shared Research Equipment (ShaRE) User Program, which is sponsored by the Scientific User Facilities Division of the Office of Basic Energy Science.

\section{Author contributions}

Z.W.P. conceived and designed the experiments. K.Y.L., X.F.L. and Z.J.G. grew graphene on CNTs. K.Y.L. and X.F.L. conducted SEM analysis. G.E. grew the aligned MWCNTs. J.H., Z.W.P. and Y.-J.C. carried out TEM imaging. S.S.X. prepared SWCNTs. Z.W.P., G.E., L.T.Z and S.S.X. contributed to graphene growth mechanisms. Z.W.P., G.E. and K.Y.L. prepared the manuscript. All authors discussed and collaborated on preparation of the manuscript.

\section{Additional information}

Supplementary information accompanies this paper at http://www.nature.com/ scientificreports

Competing financial interests: The authors declare no competing financial interests. How to cite this article: Li, K.Y. et al. Self-Assembly of Graphene on Carbon Nanotube Surfaces. Sci. Rep. 3, 2353; DOI:10.1038/srep02353 (2013)

This work is licensed under a Creative Commons AttributionNonCommercial-NoDerivs 3.0 Unported license. To view a copy of this license visit http://creativecommons.org/licenses/by-nc-nd/3.0 